\title{
Estructura de la planeación normativa como un proceso para la consolidación del Comité de Seguridad Informática en Chihuahua, Chih.
}

\author{
Structure of the Normative planning as a process of consolidation of the Information \\ Security Committee in Chihuahua, Chih.
}

\author{
CÓRDOVA-VILLEGAS, Perla Cristina†*, MEDINA-NUÑEZ, América Libertad, MORENO-OJEDA, \\ Esperanza Raquel y BARROSO-BARAJAS, Alfonso José
}

Universidad Tecnológica de Chihuahua

ID $1^{\text {er }}$ Coautor: Perla Cristina, Córdova-Villegas / ORC ID: 0000-0002-0527-4831, CVU CONACYT ID: 171313

ID $1^{\mathrm{er}}$ Autor: América Libertad, Medina-Nuñez, / ORC ID: 0000-0002-7726-8609, CVU CONACYT ID: 362150

ID $2^{\text {do }}$ Coautor: Esperanza Raquel, Moreno-Ojeda / ORC ID: 0000-0002-7231-8742, CVU CONACYT ID: 517208

ID $3^{\text {er }}$ Coautor: Alfonso José, Barroso-Barajas / ORC ID: 0000-0002-5353-5987, CVU CONACYT ID: 521749

DOI: $10.35429 /$ JCT.2019.8.3.6.10

Recibido 10 de Enero, 2019, Aceptado, 29 de Marzo, 2019

Resumen

Se presenta en estos tiempos una necesidad de fortalecer la seguridad informática de las organizaciones, lo que despierta el interés por parte del sector privado para trabajar de manera colaborativa con investigadores y educadores, en estructurar un organismo que permita que el trabajo en conjunto se pueda hacer llegar a quienes requieran asesoría en esta área. Es así como surge el Comité de Seguridad Informática, se comienza por elaborar la planeación normativa del comité antes mencionado, para poder entender y fijar sus estrategias y objetivos. Objetivos: Realizar un diagnóstico de la percepción que tiene el Comité de Seguridad Informática en formación acerca de los elementos que constituyen la planeación normativa de las organizaciones. Estructurar la misión, visión y definición de valores del Comité de Seguridad Informática en Chihuahua, Chih. Metodología: El proyecto se basa en una investigación cualitativa en donde participan cinco integrantes del Comité de Seguridad Informática a los que se les aplica la técnica de entrevista semi-estructurada para vincular el conocimiento procedimental con el contexto en que se desarrolla la actividad profesional. Contribución: Elaboración de la planeación normativa para el Comité de Seguridad Informática, estructuración de la misión, visión y definición de los valores que brindarán una guía para el establecimiento de los objetivos y estrategias.

Planeación normativa, Misión, Visión y Valores

\begin{abstract}
Nowadays there is a need to fortify the Information Security of organizations, which increase the interest of the private sector to work collaboratively with researchers and educators to structure an organism that allows to asses those who require advice in this area. This is how the Information Security Committee emerges and that is how results the need to create the sense of how it will act. Objectives: Make a diagnostic of the perception of the Information Security Committee about the elements that form the normative planning of organizations. Create the mission, vision and define of the Information Security Committee. Methodology: The project is based on a qualitative research, in which five members of the Information Security Committee a fill an semi structured interview to link the processed knowledge with the context where the professional activity is developed. Contribution: Making the normative planning for the Information Security Committee, creation of the mission, vision and the definition of the values that will provide a guide for the establishment of the objectives and strategies.
\end{abstract}

Normative planning, Mission, Vision and values

Citación: CÓRDOVA-VILLEGAS, Perla Cristina, MEDINA-NUÑEZ, América Libertad, MORENO-OJEDA, Esperanza Raquel y BARROSO-BARAJAS, Alfonso José. Estructura de la planeación normativa como un proceso para la consolidación del Comité de Seguridad Informática en Chihuahua, Chih. Revista de Tecnología Informática. 2019. 3-8: 6-10

\footnotetext{
* Correspondencia del Autor (Correo electrónico: pcordova@utch.edu.mx)

$\dagger$ Investigador contribuyendo como primer autor.
} 


\section{Introducción}

Este documento refleja el trabajo en colaboración con el Cuerpo Académico de Tecnologías de Seguridad Informática, el cual tiene como objetivo el darle al Comité la posibilidad de tener una planeación basada en los objetivos que se pretenden alcanzar, logrando que estos sean medibles y puntuales.

El siguiente artículo versa sobre la creación de la planeación normativa para el Comité antes mencionado, el cual está siendo formado por el Cuerpo Académico de Tecnologías de Seguridad Informática. Dicho Comité surge por la detección de la necesidad de contar con herramientas tecnológicas que brinden soluciones que impacten en la vida de las personas y de las instituciones, incrementando su cultura en seguridad informática, para de esta manera reducir los riesgos y vulnerabilidades que surgen por la inseguridad cibernética.

Este trabajo se desarrolla en tres etapas: primero se realiza una sesión exploratoria con los miembros del comité que permita conocer su percepción, se aplica una entrevista semiestructurada para identificar la importancia de salvaguardar la información en las organizaciones y como tercer paso se estructura la declaración de misión, visión y valores mediante el análisis y vinculación de objetivos.

\section{Justificación}

El establecer de manera correcta la misión y visión de una empresa permite definir su propósito. El Comité de Seguridad surge de la necesidad que se tiene actualmente en México; solo en el año 2018 se recibieron 1.5 millones de ataques cibernéticos al día (Forbes Staff, 2018). Pero para que este comité pueda establecer sus estrategias debe, contar con la planeación normativa adecuada.

La planeación normativa es la base del proceso administrativo de cualquier empresa o institución, para poder elaborarla es necesario realizar un diagnóstico. Una vez realizado el diagnóstico, se procede al diseño de la planeación, para poder hacerlo de manera clara y efectiva se debe conocer la información precisa de los objetivos del comité que permita su formulación basada en el "deber ser".
En esta fase se busca establecer los primeros elementos de la planeación normativa que son: la visión, con la cual se proyectan a futuro sus metas, la misión representa lo que la empresa es en la actualidad y por último, los valores que representan la identidad de la empresa.

\section{Problema}

La falta de la planeación normativa en una organización pone en riego su permanencia al carecer de estructura y objetivos en el corto mediano y largo plazo.

El carecer de una visión organizacional, limita la evaluación de costo, tiempo y resultados del comité de seguridad informática.

\section{Objetivos}

Realizar un diagnóstico de la percepción que tiene el Comité de Seguridad Informática en formación acerca de los elementos que constituyen la planeación normativa de las organizaciones.

Analizar la importancia de la protección de información digital y las vulnerabilidades a las que se enfrentan las personas y las empresas en la actualidad mediante la aplicación del método de entrevista semi-estructurada.

Estructurar la misión, visión y definición de valores mediante un procedimiento estructural y conocimiento declarativo que propicie la consolidación del Comité de Seguridad Informática en Chihuahua, Chih.

\section{Marco Teórico}

La planeación en términos generales representa el tener uno o varios objetivos establecidos y con estos van de la mano las estrategias para realizarlos de manera efectiva, en el caso de la planeación normativa se establecen parámetros para la toma de decisiones de la organización y la formulación de estrategias. (Sheen, 2018)

La base para formular las estrategias de la organización, es desarrollar la visión y la misión. En la actualidad las organizaciones deben realizar una declaración de la visión, con esta definen hacia dónde quieren dirigirse, esto sin perder de vista en qué se quiere lograr en la empresa a futuro y cómo se ve a la organización con el paso de los años. (David, 2013)

CÓRDOVA-VILLEGAS, Perla Cristina, MEDINA-NUÑEZ, América Libertad, MORENO-OJEDA, Esperanza Raquel y BARROSOBARAJAS, Alfonso José. Estructura de la planeación normativa como un proceso para la consolidación del Comité de Seguridad Informática en Chihuahua, Chih. Revista de Tecnología Informática. 2019 
La declaración de la misión le da a la organización esa claridad en el motivo o razón de ser de su creación. Al tener una Misión se tiene la descripción de los valores y los objetivos que guiarán el actuar de la organización. (David, 2013)

Para establecer los valores se debe pensar en los principios y criterios tanto éticos como morales que marcarán la pauta de las actividades de la organización; de esta manera se guía el actuar de la empresa para que sea congruente con sus objetivos. (Miklos, 1991)

Aunado a las temáticas anteriores es importante relacionar que un comité se forma con el objetivo de que un grupo de personas, que tienen el conocimiento y los medios, busquen respuesta o soluciones a necesidades que han surgido en su entorno. (Sheen, 2018)

\section{Metodología}

El proyecto se basa en investigación cualitativa en donde participan cinco integrantes del Comité de Seguridad Informática a los que se les aplica la técnica de entrevista semi-estructurada para vincular el conocimiento procedimental con el contexto en que se desarrolla la actividad profesional.

El estudio tiene como base el proceso de desarrollo de las declaraciones de misión y visión de David (2013). El punto de partida es de tipo exploratorio mediante la aplicación de un cuestionario con preguntas abiertas con la finalidad de conocer la percepción que tienen los miembros del comité respecto a la planeación normativa.

La segunda fase consiste en la aplicación de la entrevista para comprender el significado que los integrantes del comité tienen de la importancia de la seguridad informática en las organizaciones.

Para la construcción de la declaración de misión, visión y valores se convoca a los miembros del comité con la finalidad de "realizar modificaciones, adiciones y supresiones" que los vinculen con los objetivos de la organización. (David, 2013)

\section{Proceso del desarrollo de planeación normativa}

Se presentan las etapas del proceso de construcción de la planeación normativa en donde se describen citas textuales y estructuran modelos que expresan el análisis de las respuestas de los miembros del comité.

\section{Identificación de elementos clave}

Se presenta un análisis de investigación exploratoria organizada en un esquema conceptual.

\begin{tabular}{|l|l|}
\hline \multicolumn{1}{|c|}{ Componentes } & \multicolumn{1}{c|}{ Reflexión del comité } \\
\hline 1. Clientes & $\begin{array}{l}\text { Pymes e instituciones educativas } \\
\text { con manejo de tecnologías de la } \\
\text { información }\end{array}$ \\
\hline 2. Servicios & $\begin{array}{l}\text { Detección y capacitación de } \\
\text { vulnerabilidades de seguridad } \\
\text { informática. }\end{array}$ \\
\hline $\begin{array}{l}\text { 3. Alcance } \\
\text { geográfico }\end{array}$ & $\begin{array}{l}\text { Regiones más importantes del } \\
\text { estado de Chihuahua. }\end{array}$ \\
\hline $\begin{array}{l}\text { 4. Actualización } \\
\text { tecnológica }\end{array}$ & $\begin{array}{l}\text { Medianamente y en caso, se } \\
\text { solicita apoyo a especialistas. }\end{array}$ \\
\hline 5. Creencias básicas & Lo más importante es el cliente. \\
\hline 6. Prioridades éticas & $\begin{array}{l}\text { Privacidad, confianza y respeto a la } \\
\text { información del cliente. }\end{array}$ \\
\hline $\begin{array}{l}\text { 7. Ventaja } \\
\text { competitiva }\end{array}$ & $\begin{array}{l}\text { Colaboración de profesores de la } \\
\text { Universidad Tecnológica de } \\
\text { Chihuahua y empresario de } \\
\text { Globalcom. }\end{array}$ \\
\hline $\begin{array}{l}\text { 8. Preocupaciones } \\
\text { sociales }\end{array}$ & $\begin{array}{l}\text { Humanizar las tecnologías de la } \\
\text { información. }\end{array}$ \\
\hline $\begin{array}{l}\text { 9. Percepción del } \\
\text { comité a tres años }\end{array}$ & $\begin{array}{l}\text { Consolidarse sumando a miembros } \\
\text { oetras empresas e instituciones } \\
\text { educativas. }\end{array}$ \\
\hline
\end{tabular}

Tabla 1 Elementos clave para declaración de la planeación normativa

\section{Hallazgos de la entrevista semi-estructurada}

En la observación de la entrevista resalta que los miembros del comité coinciden en la relevancia que tiene actualmente el resguardo de la información en las empresas, e este respecto el maestro Alfonso Barroso describe:

"El manejo de la información siempre va a ser súper importante... incluso para los empleados pues qué pasaría con tu persona por ejemplo si toda la información de datos bancarios, tus contactos telefónicos, incluso quiénes son las personas con las que más interactúas lo conocen otras personas y organizaciones y si eso lo ponemos en un criterio malicioso ¿Qué daño podrían hacerte con esa información?" 
Entre otros hallazgos se destaca que toda persona con acceso a internet es vulnerable al robo de información privada como cuentas bancarias, direcciones, entre otros, lo que indica que las vulnerabilidades de la seguridad digital repercuten en la integridad de la vida física, y dichas debilidades se encuentran principalmente en la falta de cultura y conciencia de la sociedad en materia de seguridad informática.

A este respecto, se infiere que es imprescindible que las empresas trabajen en políticas de seguridad contemplando "actualización de hardware y software, un plan de respaldo de información, revisión periódica de procesos de seguridad y capacitación en temas de ciberseguridad" todo ello mediante la gestión oportuna de un comité de seguridad informática formalmente constituido. (Villagran Vizacarra, Ramírez Ochoa, Barba MArtínez, \& Barroso Barajas, 2018)

\section{Construcción de misión, visión y valores}

Se desarrollan tres declaraciones de misión y visión, así como siete propuestas de valores que se presentan al comité de seguridad informática con la finalidad de que todos los integrantes participen en su reconstrucción y la planeación normativa sea "un punto focal para que los individuos se identifiquen con el propósito y la dirección de la organización”. (David, 2013)

\section{Resultados}

La planeación normativa del comité de seguridad informática se constituye de la siguiente forma:

a. Misión: Ayudar a las organizaciones a proteger la información para la sistematización de procesos mediante la capacitación, asesoría e implementación de herramientas que favorezcan su permanencia en el mercado.

b. Visión: Ser el comité de seguridad informática más reconocido en el estado de Chihuahua proporcionando soluciones para el resguardo de la información de las organizaciones de forma clara e intuitiva.

c. Valores: Mejora continua, innovación, transparencia, honestidad, sinergia $\mathrm{y}$ equidad.

\section{Agradecimiento}

Agradecemos al cuerpo académico «Tecnologías de Seguridad Informática» que nos invitaron a participar con la realización de la planeación normativa del comité que están creando.

Al Ing. Ernesto García Cota dueño de la empresa «Globalcom Internacional, S.A. de C.V.» por su apoyo para la realización de este proyecto, ya que se ha involucrado en la estructura de la planeación normativa del Comité.

A los alumnos Camacho Medina Javier Eduardo, Cardoza Corral Diddier Adán, Chavira Campos Carmen Alondra, Limas Meléndez Raúl Enrique y Ruíz Ramírez Jenny Berenice por la planeación y ejecución de una entrevista semiestructurada a los miembros del Comité de Seguridad Informática.

A la Maestra Yuridia Bencomo Gaytán por su colaboración en el área de cultura organizacional.

Y a la Universidad Tecnológica de Chihuahua por el apoyo para la realización de las diversas fases y actividades del proyecto por medio del cuerpo académico «Tecnologías de seguridad Informática».

\section{Conclusiones}

Para que una organización prospere y cumpla con los objetivos planteados es indispensable que se constituya siguiendo procesos de administración estratégica. Bajo esta premisa el desarrollo de este proyecto fortalece al comité de seguridad al darle la pauta a seguir en la elaboración de estrategias y objetivos, ya que al contar con la misión, visión y los valores, el Comité tiene una guía para poder establecer cuál será su actuar.

Se sugiere al Comité de Seguridad Informática dar continuidad al fortalecimiento administrativo ahora creando la imagen corporativa de la empresa y en la medida de su desarrollo realizar una detección de necesidades en el sector empresarial. 


\section{Referencias}

David, F. R. (2013). Conceptos de administración estratégica. México: Pearson Educación.

Forbes Staff. (16 de abril de 2018). Forbes. Obtenido de Mexicanos reciben 1.5 millones de ataques cibérneticos al día: https://www.forbes.com.mx/mexicanosreciben-1-5-millones-de-ataques-ciberneticosal-dia/

Miklos, T. (1991). Planeación prospectiva: una estrategia para el diseño del futuro. México: Limusa.

Sheen, R. (2018). La cultura organizacional y su impacto en la gestión empresarial: un acercamiento a tres compañías peruanas. Lima: Fondo Editorial.

Villagran Vizacarra, D. C., Ramírez Ochoa, D. D., Barba MArtínez, C., \& Barroso Barajas, A. J. (Diciembre de 2018). Importancia de la capacitación del personal a través de una cultura de seguridad informática. Revista de Tecnologías de la Información $y$ Comunicaciones, 2(5), 11-5. Recuperado el Julio de 2019, de http://www.ecorfan.org/spain/researchjournals/ Tecnologias_de_la_Informacion_y_Comunicac iones/vol2num5/Revista_de_Tecnologia_de_la _Informacion_y_Comunicaciones_V2_N5_3.pd $\mathrm{f}$ 